\title{
Perfil epidemiológico de vítimas de violência atendidos em hospital de emergência
}

\author{
Epidemiological profile of violence victims at emergency hospital
}

Epidemiological profile of violence victims a emergency hospital

\begin{abstract}
Kalleu Leonardo Antão ${ }^{1}$, Marcelo da Silva Pinheiro ${ }^{1}$, Fernando Henrique de Oliveira Santa Maria ${ }^{1}$, Tâmyssa Simões dos Santos ${ }^{2}$, Ruth França Cizino da Trindade ${ }^{3}$, Gabriela Rodrigues Bragagnollo ${ }^{4 *}$, Marcela das Neves Guimarães², Lucyo Wagner Torres de Carvalho.
\end{abstract}

\section{RESUMO}

Objetivo: O objetivo do estudo visa analisar o perfil epidemiológico de vítimas de projetil de arma de fogo, arma branca e agressões corporais atendidas em hospital geral do estado de uma capital do nordeste brasileiro no período de 2011 a 2015. Método: Trata-se de um estudo ecológico e retrospectivo, por meio de análise dos prontuários dos pacientes atendidos no hospital. As variáveis estudadas foram: hora, local, dia e mês da ocorrência, locomoção até o hospital, idade, sexo, raça/etnia, escolaridade, escala de coma de Glasgow, conduta adotada, região corporal afetada e evolução do paciente. Resultados: Foram avaliados 5035, sendo 2984 prontuários de projetil de arma de fogo, 1276 de arma branca e 775 de agressões corporais. A faixa etária mais acometida foi de 20 a 39 anos, predominância no sexo masculino. A região corporal mais lesada foi o abdome, com projetil de arma de fogo e arma branca, $24,0 \%$ e $28,1 \%$ respectivamente. Receberam abordagem cirúrgica $84,7 \%$ e a taxa de letalidade foi 5,8\%, para arma branca. Conclusão: Esse estudo permitiu identificar a existência de risco no período noturno, além de evidenciar altas taxas de letalidade. Torna-se visível a importância do debate sobre a temática na busca de minimização dos agravos.

Descritores: Serviço Hospitalar de Emergência, Causas Externas, Perfil de Saúde, Pesquisa sobre Serviços de Saúde.

\section{ABSTRACT}

Objective: The objective of the study is to analyze the epidemiological profile of projectile victims of firearm, white weapon and corporal aggressions attended in a general hospital of the state of a capital of the Brazilian Northeast in the period of 2011 to 2015. Methods: This was a ecological and retrospective study was carried out, by analyzing the medical records of patients treated at the hospital. The variables studied were hour, place, day and month of occurrence, locomotion to the hospital, age, sex, race / ethnicity, schooling, Glasgow coma scale, adopted behavior, affected body region and patient evolution. Results: 5035 were evaluated, 2984 of which were firearm projectiles, 1276 of a white weapon and 775 of body attacks. The most affected age group was 20 to 39 years old, predominantly male. The most injured body region was the abdomen, with projectile of firearm and white weapon, $24.0 \%$ and $28.1 \%$ respectively. They received a surgical approach of $84.7 \%$ and the case fatality rate was $5.8 \%$ for the white weapon. Conclusion: This study allowed to identify the existence of risk in the night shift, in addition to evidencing high rates of lethality. The importance of the debate on the theme in the search for minimization of the diseases is made visible.

Descriptors: Emergency Service Hospital, External Causes, Health Profile, Health Services Research.

\footnotetext{
${ }^{1}$ Universidade Estadual de Ciências da Saúde de Alagoas, Maceió.

${ }^{2}$ Centro Universitário Maurício de Nassau - UNINASSAU - Maceió/Alagoas

${ }^{3}$ Universidade Federal de Alagoas - UFAL. Maceió - Alagoas.

${ }^{4}$ Escola de Enfermagem de Ribeirão Preto da Universidade de São Paulo - Ribeirão Preto - São Paulo.

*E-mail: gabriela.rodrigues.bragagnollo@usp.br
} 


\section{RESUMEN}

Objetivo: El objetivo del estudio busca analizar el perfil epidemiológico de víctimas de proyectil de arma de fuego, arma blanca y agresiones corporales atendidas en un hospital general del estado de una capital del nordeste brasileño en el período de 2011 a 2015. Métodos: Se realizó un estudio ecológico y retrospectivo, por medio de análisis de los prontuarios de los pacientes atendidos en el hospital. Las variables estudiadas fueron: hora, lugar, día y mes de la ocurrencia, locomoción hasta el hospital, edad, sexo, raza / etnia, escolaridad, escala de coma de Glasgow, conducta adoptada, región corporal afectada y evolución del paciente. Resultados: Se evaluaron 5035, siendo 2984 prontuarios de proyectil de arma de fuego, 1276 de arma blanca y 775 de agresiones corporales. El grupo de edad más acometido fue de 20 a 39 años, predominante en el sexo masculino. La región corporal más dañada fue el abdomen, con proyectil de arma de fuego y arma blanca, $24,0 \%$ y $28,1 \%$ respectivamente. Recibieron un abordaje quirúrgico $84,7 \%$ y la tasa de letalidad fue del 5,8\%, para el arma blanca. Conclusión: Este estudio permitió identificar la existencia de riesgo en el turno nocturno, además de evidenciar altas tasas de letalidad. Se hace visible la importancia del debate sobre la temática en la búsqueda de minimización de los agravios.

Palabras clave: Servicio Hospitalario de Emergencia, Causas Externas, Perfil de Salud, Investigación sobre Servicios de Salud.

\section{INTRODUÇÃO}

As causas externas, compreendidas como os acidentes e violências, ocupam posição de destaque no perfil da mortalidade de jovens (ABREU FS, et al., 2014). Constituindo, importante problema de saúde pública (MACIEL PR, et al., 2016). Dentro dos principais tipos de traumas, encontram-se a violência por projétil de arma de fogo (PAF), perfurações por arma branca (PAB), e agressões corporais, principalmente, nas grandes cidades (WAISELFISZ JJ, 2015).

Em 2014, foram registradas 61.268 internações hospitalares, destas, 29\% foram ocasionadas por PAF, que caracterizam em tentativas de morte. Nesse discurso, o que vale salientar é que as armas de fogo produzem danos, muitas vezes, irreversíveis, podendo incapacitar o indivíduo gerando altos custos para o Sistema Único de Saúde (SUS), para a Previdência Social e para as próprias famílias, por demandar cuidados do setor de saúde nos diversos níveis de complexidade, desde o pré-hospitalar até a reabilitação física e mental das vítimas (RIBEIRO AP, et al., 2017).

Mundialmente, 5 milhões de mortes anuais ocorrem em decorrência de violência, além de altas taxas de mortalidades cursam com importantes sequelas, físicas e emocionais (OMS, 2007). O PAF é o agravo que, entre as causas externas de óbito, caracteriza a $3^{\circ}$ causa de óbitos no Brasil, ficando apenas atrás de doenças do aparelho circulatório e cânceres (MALTA DC, et al., 2017).

Em países da América Latina, a posse e o uso de armas de fogo têm contribuído consideravelmente para elevar os índices de violência. A arma de fogo foi utilizada em $75 \%$ dos homicídios ocorridos em 2012 , quando mencionado países de baixa e média renda na região das Américas (RIBEIRO AP, et al., 2017).

Nessa perspectiva, a Argentina, Chile, Costa Rica e Uruguai possuem as taxas mais baixas de homicídios, enquanto que as mais altas se encontram no Brasil, México, Colômbia, El Salvador, Honduras e Venezuela. Na década de 1980, por exemplo, a Colômbia apresentava as maiores taxas de homicídios, justificada pelos fenômenos socioculturais, como a violência política e tráfico de drogas (CHAPARRO-NARVÁEZ P, et al., 2016). Em 2013, as taxas de homicídios 100.000 habitantes em alguns países da América do Sul, teve um desfecho de: Colômbia (31,7), Brasil $(28,6)$, Equador $(8,1)$, Paraguai $(7,9)$ e Uruguai $(6,4)$. No Brasil, em 2016 os homicídios alcançaram uma marca histórica de 62.517, o que equivale uma taxa de 30,3 mortes para cada 100 mil habitantes, a mesma corresponde a 30 vezes a taxa do continente europeu. Nos últimos dez anos, 553 mil pessoas perderam a vida em decorrência da violência (CERQUEIRA D, et al., 2017). 
Nesse contexto, pode-se dizer que o homicídio tem um significado social, não somente pelo fato de interromper a vida de pessoas, mas por ser reflexo dos problemas existentes na sociedade, que trazem uma íntima relação com os fatores de desigualdades, impunidade, corrupção, presença do crime organizado e da violência doméstica. Não é por acaso que as maiores taxas de homicídios ocorrem em países com baixo índice de desenvolvimento (TAVARES R, et al., 2016).

Outro causador importante de traumas são as armas brancas, que se mantêm como instrumento frequentemente utilizado, particularmente em conflitos domiciliares. Os agentes penetrantes propiciam lesões de forma direta, em função de sua trajetória e das estruturas que atravessam (SILVEIRA ES e O'DWYER G, 2017).

Ao tratar de violência, a capital de Alagoas é uma das cidades marcadas pelas características da violência urbana, encontrando-se entre as cidades mais violentas do país. Sendo enquadrada, no ano de 2012, como a capital com maiores índices de homicídios do Brasil (TRINDADE RFC, et al., 2015). Em relação aos homicídios de 2016, Alagoas ocupou uma taxa de 240,0 por 100 mil jovens homens assassinados na faixa etária de 15-29 anos, com 1.079 mortes no Estado. No país, esses dados totalizam 33.590 jovens assassinados, no qual $94,6 \%$ eram do sexo masculino. No que concerne aos homicídios por arma de fogo em Alagoas, esse chegou a 46,0\%, ocupando o segundo lugar no país. O que contabilizou 1.546 mortes somente no referido ano (CERQUEIRA D, et al., 2017).

De 1990 a 2015, as taxas de homicídio em Alagoas foram enquadradas como uma das mais altas do país $54,1 / 100$ mil habitantes (MALTA DC, et al., 2017). Alagoas também foi considerado o $4^{\circ}$ Estado com maiores taxas de mortalidade de mulheres com 9,15/100 mil hab., sendo $83,0 \%$ das mortes de mulheres brasileiras por PAF ou objetos perfurantes (GARCIA LP e SILVA GDM, 2016).

Devido à repercussão das violências, em geral, nota-se a importância do estudo dessas ocorrências para a saúde pública. Nesse contexto, estudar o perfil, analisando as causas e circunstâncias desses agravos junto à população torna-se essencial para o desenvolvimento de estratégias de promoção à saúde e prevenção.

Assim, o presente estudo tem como objetivo analisar o perfil epidemiológico de vítimas de projetil de arma de fogo, arma branca e agressões corporais atendidas em hospital geral do estado de uma capital do nordeste brasileiro no período de 2011 a 2015.

\section{MÉTODOS}

Estudo ecológico e retrospectivo, realizado por meio de análise dos prontuários dos pacientes internados em um hospital, centro de referência para urgência emergência na cidade de Maceió, Alagoas. O estudo foi aprovado pelo Comitê de Ética da Universidade Estadual de Ciências da Saúde de Alagoa, com o parecer 1.572.270, previsto de acordo com as Diretrizes e normas regulamentadoras de pesquisas envolvendo seres humanos (Resolução 466/2012, do Conselho Nacional de Saúde).

O acesso aos prontuários foi viabilizado pelo Serviço de Arquivo Médico e Estatístico (SAME) do Hospital Geral do Estado (HGE), e após assinatura de Termo de Consentimento de Utilização de dados, foram obtidos os registros de todos os pacientes internados no período de janeiro de 2011 a dezembro de 2015.

As variáveis estudadas foram: hora, local, dia e mês da ocorrência, Meio de locomoção até o hospital, idade, sexo, raça/etnia, escolaridade, escala de coma de Glasgow, tipo de conduta adotada, região corporal afetada e evolução do paciente.

Foram inclusos na pesquisa: pacientes diagnosticados com perfuração por arma de fogo, que tenham sido atendidas neste hospital durante o período em questão. Foram excluídos prontuários de casos clínicos, fichas não especificadas quanto ao tipo de atendimento e fichas ilegíveis. A primeira etapa do processo correspondeu à triagem dos prontuários de trauma, a segunda etapa é realizada com todos os prontuários de trauma, apenas selecionando os que apresentavam motivo do internamento agressões corporais por PAF e $\mathrm{PAB}$. 
Os dados coletados foram inseridos em um formulário padronizado e armazenados em uma planilha eletrônica de dados Microsoft Excel®, em dupla digitação e posterior validação dos dados. Os dados foram tabulados e grafados, na sequência os resultados foram analisados utilizando teste do Qui-quadrado, com intuito de verificar prevalências e ou associações entre a variável dependente e as independentes. $O$ programa utilizado foi o Bioestat 5.0 com alfa igual a 0,05.

\section{RESULTADOS}

No período de 01 de janeiro de 2011 a 31 de dezembro de 2015 ocorreram 2984 $(59,3 \%)$ atendimentos causados por projétil de arma de fogo e 1276 (25,3\%) por arma branca e 775 (15,4\%) agressões físicas. Os que compõem a amostra formada por 5035 prontuários avaliados no presente estudo (Tabela 1). Em todos os anos estudados a maior taxa de internação foi por PAF, com uma variação de $56,3 \%$ em 2011 a 53,1\% em 2015, entretanto, no ano de 2014 o percentual de internação foi de $65,2 \%$.

Constatou-se no período de 2011 a 2015 um decréscimo nas internações de vítimas de violência, apesar da variação verificada nas taxas de internação no período. Analisando as internações por todas as causas ocorridas no período estudado observou que as mesmas tiveram um crescimento de $55,8 \%$, sendo que as internações por agressão representaram 7,7\% do total das internações em 2011 a 3,3\% em 2015. As internações por meio de projétil de arma de fogo apresentaram uma taxa de crescimento de $-37,8 \%$, sendo a maior queda entre as causas estudadas. Também se observou uma redução nas internações de PAB de $34,5 \%$, a menor redução foi verificada na agressão com 19,8\%. Ao comparar a taxa atendimentos causados por perfuração por branca durante os anos, observou-se que foi superior em relação aos demais agravos em 2014.

Tabela 1. Distribuição dos internamentos por arma de fogo, arma branca e agressões corporais, segundo ano. Maceió, Alagoas, Brasil, 2011 - 2015.

\begin{tabular}{|c|c|c|c|c|c|c|c|}
\hline \multirow[b]{2}{*}{ Anos } & \multicolumn{2}{|l|}{ PAF } & \multicolumn{2}{|l|}{ PAB } & \multicolumn{2}{|c|}{ Agressão } & \multirow{2}{*}{$\frac{\text { Total }}{\mathbf{N}}$} \\
\hline & $\mathbf{N}$ & $\%$ & $\mathbf{N}$ & $\%$ & $\mathbf{N}$ & $\%$ & \\
\hline 2011 & 688 & 56,3 & 336 & 27,5 & 197 & 16,1 & 1221 \\
\hline 2012 & 610 & 61,0 & 253 & 25,3 & 137 & 13,7 & 1000 \\
\hline 2013 & 614 & 60,2 & 261 & 25,6 & 145 & 14,2 & 1020 \\
\hline 2014 & 644 & 65,2 & 206 & 20,9 & 138 & 14,0 & 988 \\
\hline 2015 & 428 & 53,1 & 220 & 27,3 & 158 & 19,6 & 806 \\
\hline $\begin{array}{l}\text { Taxa de Crescimento } \\
(\%)\end{array}$ & $-37,8$ & $-5,8$ & $-34,5$ & $-0,8$ & $-19,8$ & 21,5 & $-34,0$ \\
\hline
\end{tabular}

Fonte: Serviço de Arquivamento Médico (SAME) - HGE.

$\mathrm{Na}$ tabela 2 foi descrito o perfil dos atendimentos. A partir da descrição verificou-se que houve variação significativa entre o horário da ocorrência $(p<0,001)$, meses $(p<0,001)$ e meio de condução $(p<0,001)$ entre os traumas em questão. Observou-se que as maiores ocorrências das internações por arma de fogo, arma branca e agressões ocorreram no período da noite com cerca de 50,0\%, 45,0\% e 35,0\% dos casos, respectivamente.

Em relação, aos meses do ano no período em análise, a maior ocorrência das internações por projétil de arma de fogo e arma branca ocorre em janeiro e dezembro, e menor ocorrência nos meses de julho e agosto. O meio de condução mais utilizado para chegar ao hospital foi o particular/outros com aproximadamente 46,0\%, e em segundo lugar SAMU/UBS com 38,3\% de todas as internações estudadas no período (Tabela 2). 
Tabela 2. Caracterização dos internamentos por arma de arma de fogo, arma branca e agressões corporais, segundo agravo. Maceió, Alagoas, Brasil, 2011 - 2015.

\begin{tabular}{|c|c|c|c|c|c|c|c|}
\hline \multirow{2}{*}{$\begin{array}{l}\text { Horário } \\
\text { ocorrência }\end{array}$} & \multicolumn{2}{|l|}{ PAF } & \multicolumn{2}{|c|}{ PAB } & \multicolumn{2}{|c|}{ Agressão } & \multirow[t]{2}{*}{$\mathrm{P}$} \\
\hline & $\mathrm{N}$ & $\%$ & $\mathrm{~N}$ & $\%$ & $\mathrm{~N}$ & $\%$ & \\
\hline Manhã & 311 & 10,4 & 127 & 10,0 & 150 & 19,4 & \\
\hline Tarde & 639 & 21,4 & 251 & 19,7 & 193 & 24,9 & \\
\hline $\begin{array}{l}\text { Noite } \\
\text { Madrugada }\end{array}$ & $\begin{array}{l}1484 \\
550\end{array}$ & $\begin{array}{l}49,7 \\
18,4\end{array}$ & $\begin{array}{l}573 \\
325\end{array}$ & $\begin{array}{l}44,9 \\
25,5\end{array}$ & $\begin{array}{l}269 \\
163\end{array}$ & $\begin{array}{l}34,7 \\
21,0\end{array}$ & $<0,001$ \\
\hline \multicolumn{8}{|l|}{ Meses } \\
\hline Janeiro & 283 & 9,5 & 131 & 10,3 & 52 & 6,7 & \multirow{12}{*}{$<0,001$} \\
\hline Fevereiro & 243 & 8,1 & 110 & 8,6 & 61 & 7,9 & \\
\hline Março & 240 & 8,0 & 117 & 9,2 & 66 & 8,5 & \\
\hline Abril & 254 & 8,5 & 110 & 8,6 & 59 & 7,6 & \\
\hline Maio & 261 & 8,7 & 94 & 7,4 & 68 & 8,8 & \\
\hline Junho & 266 & 8,9 & 118 & 9,2 & 59 & 7,6 & \\
\hline Julho & 214 & 7,2 & 85 & 6,7 & 64 & 8,3 & \\
\hline Agosto & 215 & 7,2 & 90 & 7,1 & 73 & 9,4 & \\
\hline Setembro & 240 & 8,0 & 101 & 7,9 & 79 & 10,2 & \\
\hline Outubro & 241 & 8,1 & 107 & 8,4 & 82 & 10,6 & \\
\hline Novembro & 249 & 8,3 & 90 & 7,1 & 55 & 7,1 & \\
\hline Dezembro & 278 & 9,3 & 123 & 9,6 & 57 & 7,4 & \\
\hline \multicolumn{8}{|l|}{ Meio de condução } \\
\hline SAMU USA & 153 & 5,1 & 50 & 3,9 & 24 & 3,1 & \multirow{6}{*}{$<0,001$} \\
\hline SAMU USB & 1194 & 40,0 & 451 & 35,3 & 308 & 39,7 & \\
\hline Bombeiros & 34 & 1,1 & 15 & 1,2 & 25 & 3,2 & \\
\hline Particular & 878 & 29,4 & 307 & 24,1 & 226 & 29,2 & \\
\hline Outros & 725 & 24,3 & 453 & 35,5 & 192 & 24,8 & \\
\hline Total & 2984 & 100,0 & 1276 & 100,0 & 775 & 100,0 & \\
\hline
\end{tabular}

Fonte: Serviço de Arquivamento Médico (SAME) - HGE.

$\mathrm{Na}$ tabela 3 observou-se que houve variação significativa entre sexo $(p<0,001)$, faixa etária $(p<0,001)$, área lesada $(p<0,001)$ e GLASGOW $(p<0,001)$ entre os traumas em questão. Os casos de internações avaliados no período de 2011 a 2015, o sexo masculino foi o que mais prevaleceu em relação a quantidade de atendimentos causados por projétil de arma de fogo, arma branca e agressões com aproximadamente $92,6 \%, 87,1 \%$ e $86,5 \%$, respectivamente, sendo a razão dos sexos de nove (09) internações masculinas para cada uma (01) feminina.

Em relação faixa etária no período em análise, a maior quantidade de ocorrências de atendimentos causados por projétil de arma de fogo, arma branca e agressões aconteceu em pessoas com faixa etária entre 20-39 anos com aproximadamente $56,8 \%, 65,6 \%$ e 52,8\%, respectivamente. Ao analisar a área mais lesada no período do estudo (Tabela 3), verificou-se que a área mais acometida em pacientes por arma de fogo e arma branca foi o abdome com $24,0 \%$ e $28,1 \%$ dos casos, respectivamente. Quando se tratou de agressões a área Cabeça/Pescoço foi a mais acometida com $60,6 \%$ dos casos.

Ao avaliar os resultados obtidos através da escala de GLASGOW, verificou-se que a maioria dos pacientes vítimas de arma de fogo, arma branca e de agressões foram classificados, de acordo com a escala, como trauma leve, com 42,7\%, 45,5\% e 48,3\%, respectivamente. Entretanto, deve-se ressaltar a grande quantidade de pacientes em que neste item, não havia informação, o que chegou a 53,4\% para pacientes vítimas de arma de fogo, $51,6 \%$ de arma branca e 37,2\% de agressões (Tabela 3). 
Tabela 3 - Caracterização dos pacientes internados por arma de fogo, arma branca e agressões corporais, segundo agravo. Maceió, Alagoas, Brasil, 2011 - 2015.

\begin{tabular}{|c|c|c|c|c|c|c|c|}
\hline \multirow[t]{2}{*}{ Sexo } & \multicolumn{2}{|c|}{ PAF } & \multicolumn{2}{|l|}{ PAB } & \multicolumn{2}{|c|}{ Agressão } & \multirow[t]{2}{*}{$\mathbf{P}$} \\
\hline & $\mathrm{N}$ & $\%$ & $\mathrm{~N}$ & $\%$ & $\mathrm{~N}$ & $\%$ & \\
\hline Masculino & 2763 & 92,6 & 1111 & 87,1 & 670 & 86,5 & $<0,001$ \\
\hline Feminino & 221 & 7,4 & 165 & 12,9 & 105 & 13,5 & \\
\hline \multicolumn{8}{|l|}{ Faixa etária } \\
\hline 0 a 9 & 26 & 0,9 & 9 & 0,7 & 19 & 2,5 & \\
\hline 10 a 19 & 899 & 30,1 & 201 & 15,8 & 110 & 14,2 & \\
\hline 20 a 39 & 1696 & 56,8 & 837 & 65,6 & 409 & 52,8 & $<0,001$ \\
\hline 40 a 59 & 305 & 10,2 & 206 & 16,1 & 186 & 24,0 & \\
\hline 60 e mais & 58 & 1,9 & 23 & 1,8 & 51 & 6,6 & \\
\hline \multicolumn{8}{|l|}{ Área lesada } \\
\hline Tórax & 450 & 15,1 & 280 & 21,9 & 25 & 3,2 & \\
\hline Abdome & 717 & 24,0 & 358 & 28,1 & 31 & 4,0 & $<0,001$ \\
\hline Dorso & 48 & 1,6 & 17 & 1,3 & 7 & 0,9 & \\
\hline Cabeça/Pescoço & 413 & 13,8 & 199 & 15,6 & 470 & 60,6 & \\
\hline \multicolumn{8}{|l|}{ Membros } \\
\hline Superiores & 290 & 9,7 & 175 & 13,7 & 82 & 10,6 & \\
\hline \multicolumn{8}{|l|}{ Membros } \\
\hline Inferiores & 399 & 13,4 & 46 & 3,6 & 67 & 8,6 & \\
\hline Genitálias & 18 & 0,6 & 2 & 0,2 & 10 & 1,3 & \\
\hline \multicolumn{8}{|l|}{ Múltiplos } \\
\hline órgãos/regiões & 649 & 21,7 & 199 & 15,6 & 83 & 10,7 & \\
\hline $\mathrm{NI}$ & 0 & 0,0 & 0 & 0,0 & 0 & 0,0 & \\
\hline \multicolumn{8}{|l|}{ Glasgow } \\
\hline Leve & 1274 & 42,7 & 580 & 45,5 & 374 & 48,3 & \\
\hline Moderado & 36 & 1,2 & 17 & 1,3 & 43 & 5,5 & \\
\hline Grave & 80 & 2,7 & 21 & 1,6 & 70 & 9,0 & $<0,001$ \\
\hline $\mathrm{NI}$ & 1594 & 53,4 & 658 & 51,6 & 288 & 37,2 & \\
\hline Total & 2984 & 100,0 & 1276 & 100,0 & 775 & 100,0 & \\
\hline
\end{tabular}

Fonte: Serviço de Arquivamento Médico (SAME) - HGE.

Tabela 4. Caracterização dos desfechos dos internados por arma de fogo e arma branca, segundo agravo. Maceió, Alagoas, Brasil, 2011 - 2015.

\begin{tabular}{|c|c|c|c|c|c|c|c|}
\hline \multirow{2}{*}{ Tratamento } & \multicolumn{2}{|c|}{ PAF } & \multicolumn{2}{|c|}{ PAB } & \multicolumn{2}{|c|}{ Agressão } & \multirow[t]{2}{*}{$\mathbf{P}$} \\
\hline & $\mathbf{N}$ & $\%$ & $\mathbf{N}$ & $\%$ & $\mathbf{N}$ & $\%$ & \\
\hline Clínico & 802 & 26,9 & 195 & 15,3 & 505 & 65,2 & \\
\hline Cirúrgico & 2182 & 73,1 & 1081 & 84,7 & 270 & 34,8 & $<0,001$ \\
\hline $\mathrm{NI}$ & 0 & 0,0 & 0 & 0,0 & 0 & 0,0 & \\
\hline \multicolumn{8}{|l|}{ Evolução } \\
\hline Alta & 1897 & 63,6 & 929 & 72,8 & 602 & 77,7 & \\
\hline Transferência & 690 & 23,1 & 273 & 21,4 & 85 & 11,0 & \\
\hline Óbito & 397 & 13,3 & 74 & 5,8 & 88 & 11,4 & $<0,001$ \\
\hline $\mathrm{NI}$ & 0 & 0,0 & 0 & 0,0 & 0 & 0,0 & \\
\hline Total & 2984 & 100,0 & 1276 & 100,0 & 775 & 100,0 & \\
\hline
\end{tabular}

Fonte: Serviço de Arquivamento Médico (SAME) - HGE. 
A partir da descrição identificou-se que houve variação significativa entre Tipo de tratamento $(p<0,001)$ e evolução $(p<0,001)$, entre os traumas em questão. Para pacientes vítimas de arma de fogo e arma branca, verificou-se que o tratamento mais predominante foi o cirúrgico com aproximadamente $73,0 \%$ e $85,0 \%$ dos casos, respectivamente. Já a maioria dos pacientes internados vítimas de agressão tiveram tratamento clínico, 65,2\% dos casos.

Para pacientes vítimas de arma de fogo, arma branca e agressão verificou-se que a alta foi a que mais prevaleceu com $63,6 \%, 72,8 \%$ e $77,7 \%$ dos casos, respectivamente. A taxa de óbito para vítimas de arma de fogo, agressão, arma branca foi de 13,3\%, 11,4\% e 5,8\% dos casos, respectivamente (Tabela 4).

\section{DISCUSSÃO}

A análise dos dados mostra que houve variação com relação a quantidades de internamentos nos anos estudados. Houve diminuição considerável dos agravos em comparação 2011 e 2015. Quanto a faixa etária, a mais acometida nos pacientes atendidos na unidade em todo período estudado foi de 20 a 39 anos representando no PAF 1696 (56,8\%), nas PAB 837 (65,6\%), e agressões 409 (52,8\%). O perfil etário identificado nos prontuários foi semelhante aos achados descritos na literatura, a qual constatou porcentagens semelhantes em Teresina, Jequié-BA e Goiânia (ABREU FS, et al., 2014; FALLIS A, 2013; MACIEL PR, et al., 2016). Em outro estudo realizado em Maceió a faixa etária foi de 15 a 39 anos, essa faixa etária tem sido apontada como a principal relacionada a vítimas dos agravos em questão, principalmente, sendo associado ao comportamento de risco e exposição a qual os jovens estão submetidos (ABREU FS, et al., 2014; TRINDADE RFC, et al., 2015).

O sexo masculino predominou tanto no que diz respeito às causas externas de uma forma geral, quanto em relação às agressões específicas. Em um estudo sobre o perfil das vítimas com ferimentos por projéteis de arma de fogo e dos custos assistenciais em um hospital da Rede Viva Sentinela o sexo masculino representou 94,7\% (MACIEL PR, et al., 2016). Em avaliação do perfil das vítimas fatais por arma de fogo no município de João Pessoa, a mortalidade por PAF chegou a representar $95 \%$ dos casos no sexo masculino (JÚNIOR JFB, et al., 2016). Dados do Sistema de Informações sobre Mortalidade (SIM), da Secretaria de Vigilância em Saúde do Ministério da Saúde (SVS/MS), evidenciou que 79,6\% da mortalidade de jovens com idade entre 15 e 29 anos eram do sexo masculino (NEVES ACM e GARCIA LP, 2016).

Quanto ao meio de locomoção até o hospital, mais de 50\% das vítimas foram deslocadas em carros particulares ou outros veículos. Diferindo de um estudo realizado no Rio de Janeiro, a qual as vítimas foram trazidas por ambulâncias do Corpo de Bombeiros (SILVEIRA ES e O'DWYER G, 2017).

Em relação ao horário de atendimento demostrou que existe predominância para todos os agravos (PAF, $\mathrm{PAB}$ e agressões) no período noturno, que compreende entre as 18:00 e 23h59. Estes dados convergem com outros estudos, que associam o horário de diversão, com o período de maior consumo de bebidas e drogas, e estes podem favorecer o envolvimento e/ou a exposição em situações de violência que levem à agressão (SILVEIRA ES e O'DWYER G, 2017; MASCARENHAS MDM, et al., 2015). Defendendo esta hipótese, na pesquisa VIVA Inquérito foi detectado que $44,1 \%$ dos pacientes atendidos por causas violentas, apresentavam prevalência de consumo de bebida alcoólica (MASCARENHAS MDM, et al., 2015).

A partir da interpretação dos dados, evidenciou que a maioria das vítimas apresentava lesão única por PAF de 2.335 (78,3\%), e múltiplas em 649 (21,7\%) dos casos. Ainda assim, nos PAF, predominaram o abdome 717 (24\%) e o tórax 450 (15,1\%). Estando em consonância com outros estudos (MACIEL PR, et al., 2016; GARCIA LP e SILVA GDM, 2016). A lesão única também foi mais presente nas PAB 1077 (84,4\%), no entanto, foi identificado em um dado estudo, maior porcentagem em pacientes que possuíam lesões múltiplas. Sendo as regiões mais acometidas abdome $358(28,1 \%)$ e o tórax $280(21,9 \%)$ (TRINDADE RFC e CORREIA MAA, 2015).

No que diz respeito a evolução dos casos estudados, observou-se que nos PAF 1897 (63,6\%) evoluíram com alta hospitalar, o êxito letal representou $397(13,3 \%)$ e $690(63,6 \%)$ dos pacientes transferidos para outras 
unidades de saúde. Nos traumas por PAB, receberam alta 929 (72,8\%), $74(5,8 \%)$ óbitos e $273(21,4 \%)$ transferidos. Dados convergentes com trabalho que analisaram atendidos por PAF e PAB, o qual apresentou taxa de óbitos de 7,7\% (ABREU FS, et al., 2014), e em Goiânia, foi de 9,7\% (MACIEL PR, et al., 2016). Na cidade de Maceió no ano de 2012 a taxa de mortalidade chegou a 16,4\% (FALLIS A, 2013). Em João Pessoa, as vítimas fatais de PAF, também estavam entre 18 e 35 anos, sendo $43,0 \%$ dos óbitos com lesões de cabeça e, $30,0 \%$ com o tórax, caracterizando as duas principais regiões correlacionadas com mortalidade (JÚNIOR JFB, et al., 2016).

Os dados referentes à mortalidade podem não refletir a realidade com relação aos agravos em questão, levando em consideração o número de transferências para outros hospitais, de rede pública ou privada do estado, os quais o hospital não possui controle sobre informações posteriores a transferência.

Os resultados apontam que a fase mais suscetível para o acometimento dos agravos em questão é a juventude, predominantemente do sexo masculino. Esta pesquisa levantou aspectos relevantes sobre o perfil epidemiológico do trauma, os quais podem fomentar a implantação a elaboração de políticas para o enfrentamento dos agravos em questão, tendo em vista que se trata de problemas que podem acometer toda sociedade. É importante ressaltar alguns tópicos como a ocorrência, em sua maioria, no turno noturno.

Para entender o cenário e os indivíduos que estão envolvidos na prática de homicídios no mundo, faz-se necessário traçar linhas gerais de intervenção do Estado, o que permite observar que alguns países trazem uma carga de abordagem mais punitiva, repressora e de maior controle sobre os cidadãos, da mesma forma que se evidencia países com uma persecução penal maximizada, com as penas voltadas para os mais variados crimes, com o intuito de diminuir ou desestimular os delitos. No entanto, outros países adotam uma postura de políticas e intervenções preventivas, atuando nas causas determinantes dos crimes (ROCHA PL, 2015).

As limitações encontradas no estudo foram a carência de algumas informações, como: etnia e escolaridade dos pacientes, informações estas que importantes no viés da epidemiologia. Outra limitação foi, principalmente, de ordem física, em que os prontuários eram armazenados em caixas de papel, e sem nenhuma subdivisão, letras ilegíveis ou incompreensíveis.

\section{CONCLUSÃO}

Esse estudo permitiu identificar a existência de risco vítimas da violência no período noturno, além de evidenciar altas taxas de letalidade, que foram similares quanto a violência por arma de fogo, arma branca e agressões. Os resultados apontam que a fase mais suscetível para o acometimento dos agravos é a juventude, que é um dado preocupante, pois, estes são responsáveis pela produção econômica do país. Em relação à distribuição mensal, ficou comprovado que os meses foram homogêneos para a quantidade de ocorrência dos agravos, no entanto, em janeiro e dezembro, foram registrados o maior número de casos, e menor nos meses de julho e agosto.

Há, portanto, necessidade de pesquisas nessa área, tendo em vista que a violência é um problema que afeta todos estratos sociais, e de medidas intersetoriais que trabalhem a prevenção dos agravos para que possam promover de forma direta e indireta a diminuição do agravo. Esta pesquisa levantou aspectos relevantes sobre o perfil epidemiológico do trauma, os quais podem fomentar a implantação e a elaboração de políticas para o enfrentamento dos agravos em questão, tendo em vista que se tratam de problemas que podem acometer toda sociedade. Assim sendo, torna-se visível a importância do debate sobre a temática na busca de minimização dos agravos.

\section{REFERÊNCIAS}

1. ABREU FS, et al. Perfil epidemiológico das agressões por arma de fogo e arma branca no interior da Bahia. Revista Saúde.Com, 2014; 10(4):360-367.

2. CERQUEIRA D, et al. Atlas da violência 2017. Brasília: IPEA, 2017; 69p. 
3. CHAPARRO-NARVÁEZ P, et al. Mortality by homicides in Colombia, 1998-2012. Biomédica [Internet], 2016; 36(4):572-582.

4. FALLIS A. Perfil das vítimas de trauma atendidas pelo serviço de atendimento móvel de urgência. J. Chem. Inf. Model., 2013; 53(9):1689-99.

5. GARCIA LP, SILVA GDM. Mortalidade de mulheres por agressões no Brasil: perfil e estimativas corrigidas (2011 - 2013). Brasília: IPEA, 2016; 42p.

6. JÚNIOR JFB, et al. Perfil das vítimas fatais por arma de fogo no município de João Pessoa. Revista de Ciências da Saúde Nova Esperança, 2016; 14(1):15-26.

7. MACIEL PR, et al. Estudo descritivo do perfil das vítimas com ferimentos por projéteis de arma de fogo e dos custos assistenciais em um hospital da Rede Viva Sentinela. Epidemiologia e Serviços Saúde [Internet], 2016; 25(3):607-16.

8. MALTA DC, et al. Mortalidade e anos de vida perdidos por violências interpessoais e autoprovocadas no Brasil e Estados: análise das estimativas do Estudo Carga Global de Doença, 1990 e 2015. Revista Brasileira de Epidemiologia [Internet], 2017; 20(suppl 1):142-56.

9. MASCARENHAS MDM, et al. Emergency room visits due to external causes and alcohol consumption - Capitals and the Federal District, Brazil, 2011. Ciência \& Saúde Coletiva [Internet], 2015; 20(4):1037-46.

10. NEVES ACM, GARCIA LP. Mortalidade de jovens brasileiros: perfil e tendências no período $2000-2012$. Epidemiologia e Serviços de Saúde [Internet], 2015; 24(4):595-606.

11. OMS. Preventing injuries and violence: a guide for ministries of health. Who [Internet]. 2007;1-36.

12. RIBEIRO AP, et al. Lesões provocadas por armas de fogo atendidas em serviços de urgência e emergência brasileiros. Ciência \& Saúde Coletiva [Internet], 2017; 22(9):2851-60.

13. ROCHA PL. Análise das taxas de homicídios, indicadores de desenvolvimento humano e políticas de segurança pública: o caso do Chile no contexto latino-americano. Trabalho de Conclusão de Curso (Graduação em Direito) - Faculdade de Direito. Universidade Federal de Brasília, Brasília, 2015; 53 p.

14. SILVEIRA ES, O'DWYER G. Centro de Trauma: modelo alternativo de atendimento às causas externas no estado do Rio de Janeiro. Saúde em Debate [Internet], 2017; 41(112):243-54.

15. TAVARES R, et al. Homicídios e vulnerabilidade social. Ciência \& Saúde Coletiva [Internet], 2016;21(3):923-34.

16. TRINDADE RFC, CORREIA MAA. Perfil epidemiológico das vítimas de armas branca e de fogo em um hospital de emergência. Revista de Enfermagem e Atenção à Saude, 2015; 4(1):55-64.

17. TRINDADE RFC, et al. Map of homicides by firearms: Profile of the victims and the assaults. Revista da Escola de Enfermagem, 2015; 49(5):748-55.

18. WAISELFISZ JJ. Mapa da Violência 2015: Mortes Matadas por Armas de Fogo. Brasília: Unesco, 2015; 112p. 